\title{
WEIGHT FUNCTIONS FOR A CLASS OF LIAPUNOV FUNCTIONS IN THE PLANE*
}

\author{
By \\ LARRY R. ANDERSON AND SARAH E. DUCICH \\ Whitman College, Walla Walla
}

\begin{abstract}
In this paper we give a class of weight functions which generate Liapunov functions for a general second-order differential system. In the special case of a Lienard equation we give conditions under which these weight functions may be chosen so as to improve certain known estimates of regions of asymptotic stability. The procedure is applied to a well-known equation and new estimates are obtained.
\end{abstract}

1. Introduction. Two recent papers $[1,2]$ consider the problem of finding weight functions $\theta(x, y)$ such that functions

$$
V_{\theta}(x, y)=\int_{h(x)}^{y} \theta(x, s) f(x, s) d s-\int_{0}^{x} \theta[s, h(s)] g[s, h(s)] d s
$$

are Liapunov functions for a differential system $\dot{x}=f(x, y), \dot{y}=g(x, y)$, where $f[x, h(x)] \equiv 0$. These papers show that in many cases there are many admissible values of $\theta$. In [1] it is shown that, in the special case of the van der Pol equation, one can choose $\theta$ so as substantially to improve the estimate of the region of asymptotic stability of an isolated critical point over a well-known estimate.

In this paper we generalize the work done in [1]. In particular, we consider weight functions of the form

$$
\theta(x, y)=\lambda_{1} \int_{0}^{y} \phi(s) d s+\lambda_{2} \int_{0}^{x} r(s, 0) d s
$$

for the system

$$
\dot{x}=\phi(y), \quad \dot{y}=r(x, y),
$$

and show that in a large class of cases, functions $V_{\theta}$ provide Liapunov functions for systems (1.3). In the special case of a Lienard equation we give general conditions under which one can choose constants $\lambda_{1}, \lambda_{2}$ in (1.2) so as to improve the estimate of the region of asymptotic stability over well-known estimates that may be obtained by using $\theta$ with $\lambda_{1}=\lambda_{2}$. Finally, we apply the method to an equation that appears extensively in the literature $[2,3,4,5]$ and obtain improved estimates.

As was pointed out in [1], an unsolved problem is the general problem of finding $\theta$ so as to maximize the estimate of the region of asymptotic stability among admissible values

\footnotetext{
* Received May 22, 1979; revised version received April 1, 1980.
} 
of $\theta$. This problem remains unsolved. However, the procedure found in Secs. 3 and 4 of this paper does provide a method for maximization over a subclass of admissible values of $\theta$.

2. A general class of Liapunov functions. We consider the system

$$
\dot{x}=\phi(y), \quad \dot{y}=r(x, y),
$$

and assume $\phi(y)$ and $r(x, y)$ are of class $C^{\prime}$ in an open rectangle $R$ containing the origin and that the origin is the only equilibrium point of (1.1) in $R$. We further assume that $r(x, 0)$ is of class $C^{\prime}$ for all $x$. We state the following lemma, which is well known.

Lemma. Suppose $r(x, 0) \in C^{\prime}(-\infty, \infty)$ and $r(0,0)=0$. Then there exist functions $w(x)$ and $A(x)$, continuous on $R$, such that $r(x, 0)=x w(x)$ and $-\int_{0}^{x} r(s, 0) d s=x^{2} A(x)$.

Proof. Let $\gamma(t)=r(t x, 0)$. Then $\gamma(1)-\gamma(0)=r(x, 0)=x \int_{0}^{1} r_{x}(x t, 0) d t$. If we define $w(x)=\int_{0}^{1} r_{x}(x t, 0) d t$ and let $A(x)=x^{-2} \int_{0}^{x} s w(s) d s$ for $x \neq 0$, then $\lim _{x \rightarrow 0} A(x)=$ $\lim _{x \rightarrow 0}(2 x)^{-1}(x w(x))=w(0) / 2=r_{x}(0,0) / 2$. This proves the lemma.

Now let

$$
P(x)=-\int_{0}^{x} r(s, 0) d s=x^{2} A(x), \quad I(y)=\int_{0}^{y} \phi(s) d s .
$$

We define $\theta(x, y)=\lambda_{1} I(y)+\lambda_{2} P(x)$ where $\lambda_{1}$ and $\lambda_{2}$ are to be determined. From (1.1) and (1.2) we obtain

$$
V_{\theta}(x, y)=\frac{\lambda_{1} I^{2}(y)}{2}+\lambda_{2} I(y) P(x)+\frac{\lambda_{2}}{2} \frac{P^{2}(x)}{2} .
$$

In order for $V_{\theta}$ to be positive definite, it is enough to assume that $\lambda_{1}>0, \lambda_{2}>0$.

Computing $\dot{V}_{\theta}$ along the trajectories of (2.1), we obtain

$$
\begin{aligned}
\dot{V}_{\theta}(x, y)= & \phi(y) \lambda_{2} I(y)[-r(x, 0)]+\phi(y) \lambda_{2}[-P(x)] r(x, 0) \\
& +\lambda_{1} I(y) \phi(y) r(x, y)+\lambda_{2} \phi(y) P(x) r(x, y), \\
= & \lambda_{1} \phi(y) I(y)[r(x, y)-r(x, 0)]+\phi(y) I(y)\left(\lambda_{1}-\lambda_{2}\right) r(x, 0) \\
& +\lambda_{2} \phi(y)[r(x, y)-r(x, 0)] P(x), \\
= & y D(x, y)\left[\frac{\phi(y) I(y)}{y^{3}} \lambda_{1} y^{2}+\frac{\phi(y) I(y) w(x)\left(\lambda_{1}-\lambda_{2}\right)}{y^{2} D(x, y)} x y+\frac{\phi(y) A(x)}{y} \lambda_{2} x^{2}\right],
\end{aligned}
$$

where $w(x)$ and $A(x)$ are given in the lemma and

$$
D(x, y)=r(x, y)-r(x, 0)=\int_{0}^{y} r_{y}(x, s) d s .
$$

Note that $y D(x, y)<0$ for $y \neq 0$. Therefore, if $\dot{V}_{\theta}$ is to be negative semidefinite, the quantity above in square brackets must be positive semidefinite. Since this quantity is a quadratic form with variable coefficients, it will be positive semidefinite if its discriminant is nonpositive. In this case, for $y \neq 0$, the discriminant equals

$$
\frac{\phi^{2}(y) I(y)}{y^{6}}\left[\frac{I(y) w^{2}(x)\left(\lambda_{1}-\lambda_{2}\right)^{2}}{\left[y^{-1} D(x, y)\right]^{2}}-4 y^{2} \lambda_{1} \lambda_{2} A(x)\right] \text {. }
$$


Thus the discriminant is nonpositive if

$$
\frac{\left(\lambda_{1}-\lambda_{2}\right)^{2}}{\lambda_{1} \lambda_{2}} \leq \frac{4 A(x)[D(x, y)]^{2}}{w^{2}(x) I(y)}
$$

which is equivalent to

$$
\frac{\lambda_{1}}{\lambda_{2}}+\frac{\lambda_{2}}{\lambda_{1}} \leq 2+\frac{4 A(x)[D(x, y)]^{2}}{w^{2}(x) I(y)} .
$$

THEOREM 1. Assume that $r(x, y)$ satisfies

(i) $r(x, 0) \in C^{\prime}(-\infty, \infty)$,

(ii) $x r(x, 0)<0$ for all $x \neq 0$,

(iii) $r_{y}(x, y)<0$ neighboring $(0,0)$,

(iv) $r(0,0)=0$.

Further assume $\phi(y)$ is continuous neighboring the origin and locally satisfies $y \phi(y)>0$ for $y \neq 0$. Then there exist positive constants $\lambda_{1}$ and $\lambda_{2}$ such that the function

$$
V_{\theta}(x, y)=\frac{\lambda_{1} I^{2}(y)}{2}+\lambda_{2} I(y) P(x)+\frac{\lambda_{2} P^{2}(x)}{2}
$$

is a Liapunov function for $(2.1)$. That is, $V_{\theta}(x, y)$ is positive definite and $\dot{V}_{\theta}(x, y) \leq 0$ neighboring the origin.

Proof. Choose $\lambda_{1}=\lambda_{2}$. The result follows from the preceding analysis.

With $\lambda_{1}=\lambda_{2}>0$, the function (2.3) becomes $\lambda_{1} V^{2} / 2$, where $V=I(y)+P(x)$ is the function given in [2]. In this case the estimate of the stability region given by (2.3) is the same as the estimate given by $V$. There are, however, many general cases in which constants $\lambda_{1}$ and $\lambda_{2}$ may be chosen so as to improve the estimate given by $V$. One such general case will be explored thoroughly in the following section.

3. Estimates for Lienard equations. We consider in this section the equation

$$
\ddot{y}+f(y) \dot{y}+g(y)=0
$$

and the associated equivalent system

$$
\dot{x}=g(y), \quad \dot{y}=-x-F(y),
$$

where $F(y)=\int_{0}^{y} f(s) d s$. If the conditions of Theorem 1 hold, then the origin is asymptotically stable. Further, the global behavior of (3.2) and the estimates by (2.3) depend heavily upon the behavior of $g$ and $F$. We consider the case where (3.2) has an asymptotically stable equilibrium point and a single neighboring unstable equilibrium point. A separate analysis may be given under different conditions; for example, the case where the origin is a unique equilibrium point surrounded by a unique limit cycle (see [1]).

For this case we assume the following:

(i) $g(y)>0$ for $y>0$ and $g(y) \rightarrow \infty$ as $y \rightarrow \infty$,

(ii) $g\left(y_{0}\right)=0$ for some $y_{0}<0$,

(iii) $g(y)<0$ for $y \in\left(y_{0}, 0\right)$,

(iv) $g(y)>0$ for $y<y_{0}$ and $g(y) \rightarrow \infty$ as $y \rightarrow-\infty$,

(v) $f(y)>0$ for all $y$, and

(vi) $f, g \in C^{\prime}(-\infty, \infty)$. 
To consider the regions of asymptotic stability we state the following theorem which has been proved elsewhere [4].

THEOREM 2. Suppose the system

$$
\dot{X}=f(X)
$$

has an isolated equilibrium point at the origin and suppose further that there exists a function $V(X)$, with $V(0)=0$, of class $C^{\prime \prime}$ in $E_{n}$, which is positive definite neighboring the origin and has a finite number of critical points, finite or infinite. Then, there is a positive critical value $k$ (possibly infinite) of $V$ such that for each number $a$ on the interval $0<a<k$, the equations $V=a$ define closed manifolds with $(0) \in\{V<a\}$. If, throughout $\{V<a\}, \dot{V} \leq 0$, the set $\{V<k\}$ bounds a region of stability of the origin.

We now compare the estimates given by $V_{\theta}$ in $(2.3)$ and

$$
V(x, y)=\int_{0}^{y} g(s) d s+x^{2} / 2 .
$$

Assuming (3.3), the only critical values are $\lambda_{1}\left(\int_{0}^{y_{0}} g(s) d s\right)^{2} / 2$ and $\int_{0}^{y_{0}} g(s) d s$, respectively. Therefore, letting $k=\int_{0}^{y_{0}} g(s) d s$,

$$
\frac{\lambda_{1}\left(\int_{0}^{y} g(s) d s\right)^{2}}{2}+\frac{\lambda_{2} x^{2} \int_{0}^{y} g(s) d s}{2}+\frac{\lambda_{2} x^{4}}{8}=\frac{\lambda_{1} k^{2}}{2} \quad\left(y \geq y_{0}\right)
$$

encloses a region of stability if it lies in a domain $B$ containing the origin such that in $B$, for $y \neq 0$,

$$
\lambda_{1} \lambda_{2}^{-1}+\lambda_{2} \lambda_{1}^{-1} \leq 2+2 F^{2}(y)\left[\int_{0}^{y} g(s) d s\right]^{-1} .
$$

Indeed, if (3.4) lies in such a region $B$, then the interior of (3.4) is a region of asymptotic stability. To see this, note first that $f(y)>0$ for all $y$, so that by Green's theorem (3.2) has no nontrivial periodic solutions. It is then an immediate consequence of the PoincareBendixson theorem (see [6, p. 184]) that the positive limit set of any solution starting inside (3.4) is either the origin or contains the origin and $\left(-F\left(y_{0}\right), y_{0}\right)$. But any trajectory starting inside (3.4) will not leave the interior of some $V_{\theta}=a<\lambda_{1} k^{2} / 2$ (see [4]) and hence cannot approach $\left(-F\left(y_{0}\right), y_{0}\right)$ along some sequence $\left\{t_{n}\right\} \rightarrow \infty$.

Since the determination of the constants $\lambda_{1}$ and $\lambda_{2}$ is dependent upon their ratio, we let $\lambda=\lambda_{2} \cdot \lambda_{1}^{-1}$. Then (3.4) becomes

$$
\frac{\left(\int_{0}^{y} g(s) d s\right)^{2}}{2}+\frac{\lambda x^{2} \int_{0}^{y} g(s) d s}{2}+\frac{\lambda x^{4}}{8}=\frac{k^{2}}{2} \quad\left(y \geq y_{0}\right)
$$

and condition (3.5) becomes

$$
\lambda+\lambda^{-1} \leq 2+2 F^{2}(y)\left[\int_{0}^{y} g(s) d s\right]^{-1} .
$$

To compare the estimates of the regions of asymptotic stability, we assume that for $y \neq 0$

$$
2 F^{2}(y)\left[\int_{0}^{y} g(s) d s\right]^{-1} \geq \alpha>0
$$


inside a strip $-\delta_{1}<y<\delta_{2}$ and that the curve (3.6) lies wholly within this strip. Then we choose $\lambda$ such that

$$
\lambda+\lambda^{-1} \leq 2+\alpha .
$$

Next, solve $V(x, y)=k$ for $x^{2}$. Also, solve for $x^{2}$ in (3.6). We obtain numbers $x_{1}^{2}$ and $x_{2}^{2}$, respectively. Using the notation of $(2.2), x_{1}^{2}=2(k-I(y))$ and

$$
x_{2}^{2}=2\left(-I(y)+\left[k^{2} \lambda^{-1}+I^{2}(y)\left(1-\lambda^{-1}\right)\right]^{1 / 2}\right) .
$$

Then, if $x_{1}^{2}$ and $x_{2}^{2}$ are both defined at $y$, we have

$$
\omega(\lambda, y)=x_{2}^{2}-x_{1}^{2}=\frac{2\left(1-\lambda^{-1}\right)(I(y)-k)(I(y)+k)}{k+\left[k^{2} \lambda^{-1}+I^{2}(y)\left(1-\lambda^{-1}\right)\right]^{1 / 2}} .
$$

If $0<\lambda<1$, we have $\omega(\lambda, y)>0$ and $\partial \omega / \partial \lambda<0$ whenever $I(y)<k$.

We summarize the above analysis.

Theorem 3. Let conditions (3.3) hold. Assume that the curve (3.6) lies in a strip $-\delta_{1}<y<\delta_{2}$ and that (3.8) holds in this strip. If $0<\lambda<1$ and $\lambda+\lambda^{-1} \leq 2+\alpha$, then the estimate of the region of asymptotic stability given by (3.6) wholly contains the estimate given by $V(x, y)=k$. With the above procedure, maximal improvement over the estimate $V(x, y)=k$ is obtained by choosing $\lambda$ such that $0<\lambda<1$ and $\lambda+\lambda^{-1}=2+\alpha$.

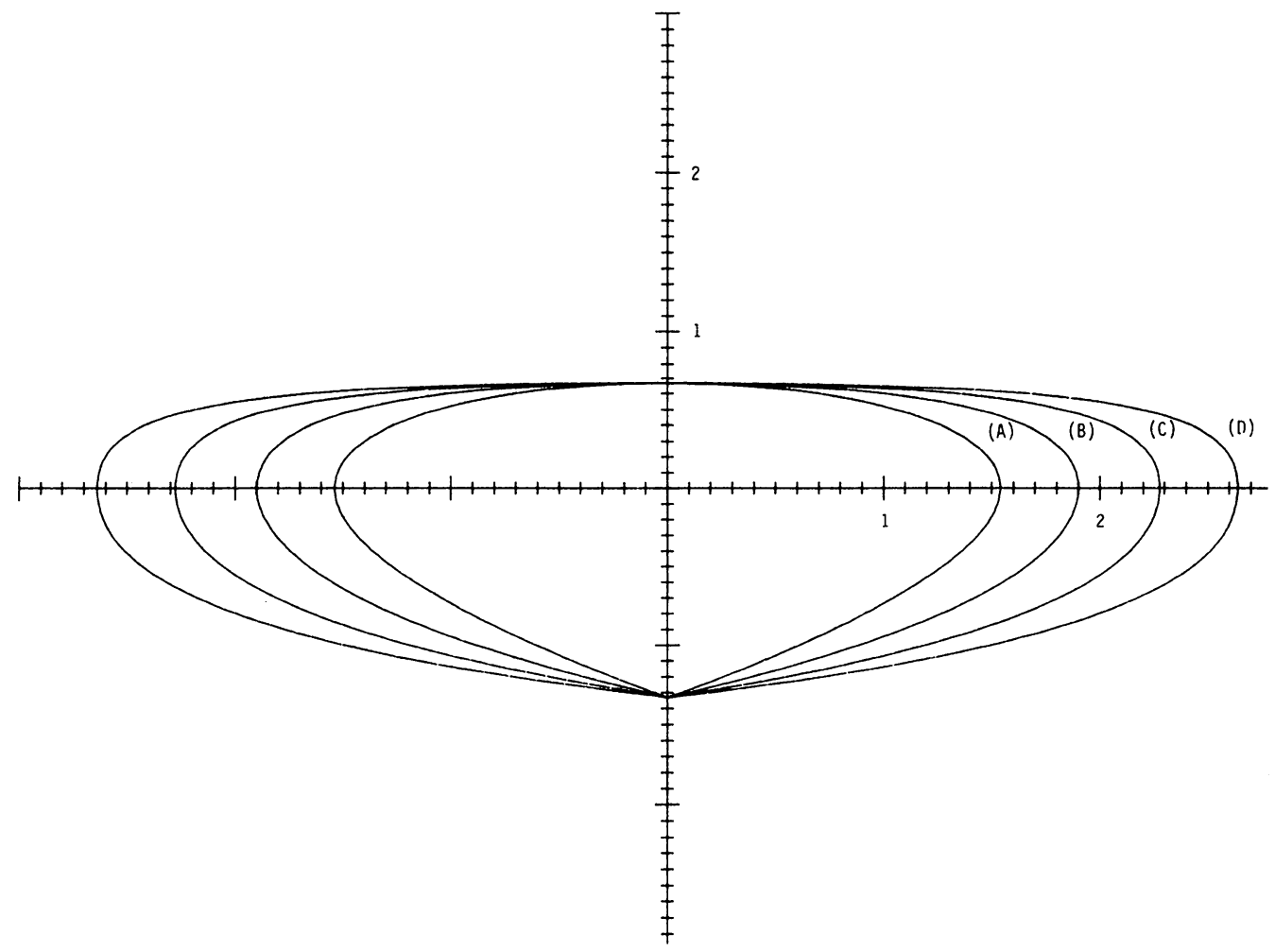

Fig. 1. (A) curve (4.6), $b=2$; (B) curve (4.5), $a=1, b=2$; (C) curve (4.5), $a=2, b=2$; (D) curve (4.5), $a=3$, $b=2$. 
4. Example. We now consider the equation

$$
\ddot{y}+a \dot{y}+2 b y+3 y^{2}=0
$$

where $a>0, b>0$. This equation has been studied extensively (e.g. see [2, 3 or 4$]$ ). This equation is equivalent to the system

$$
\dot{x}=2 b y+3 y^{2}, \quad \dot{y}=-x-a y .
$$

For this system the equation (3.6) becomes

$$
\frac{\left(b y^{2}+y^{3}\right)^{2}}{2}+\lambda \frac{x^{2}}{2}\left(b y^{2}+y^{3}\right)+\lambda \frac{x^{4}}{8}=\left[\frac{4 b^{3}}{27}\right]^{2} \cdot \frac{1}{2} \quad\left(y \geq \frac{-2 b}{3}\right) .
$$

This curve encloses a region of asymptotic stability for (4.2) if it lies in a strip $-\delta_{1}<y<\delta_{2}$ such that in this strip

$$
\lambda+\lambda^{-1} \leq 2+2 a^{2}(b+y)^{-1} .
$$

From (4.3) note that $-2 b / 3 \leq y \leq b / 3$. Therefore, if $\lambda+\lambda^{-1} \leq 2+3 a^{2}(2 b)^{-1}$, then (4.4) holds. Solving this inequality for $\lambda$ and choosing $0<\lambda<1$ and $\lambda+\lambda^{-1}=2+$ $3 a^{2}(2 b)^{-1}$, we obtain

$$
\lambda_{0}=2\left[2+\frac{3 a^{2}}{2 b}+\left(\left[2+\frac{3 a^{2}}{2 b}\right]^{2}-4\right)^{1 / 2}\right]^{-1}
$$

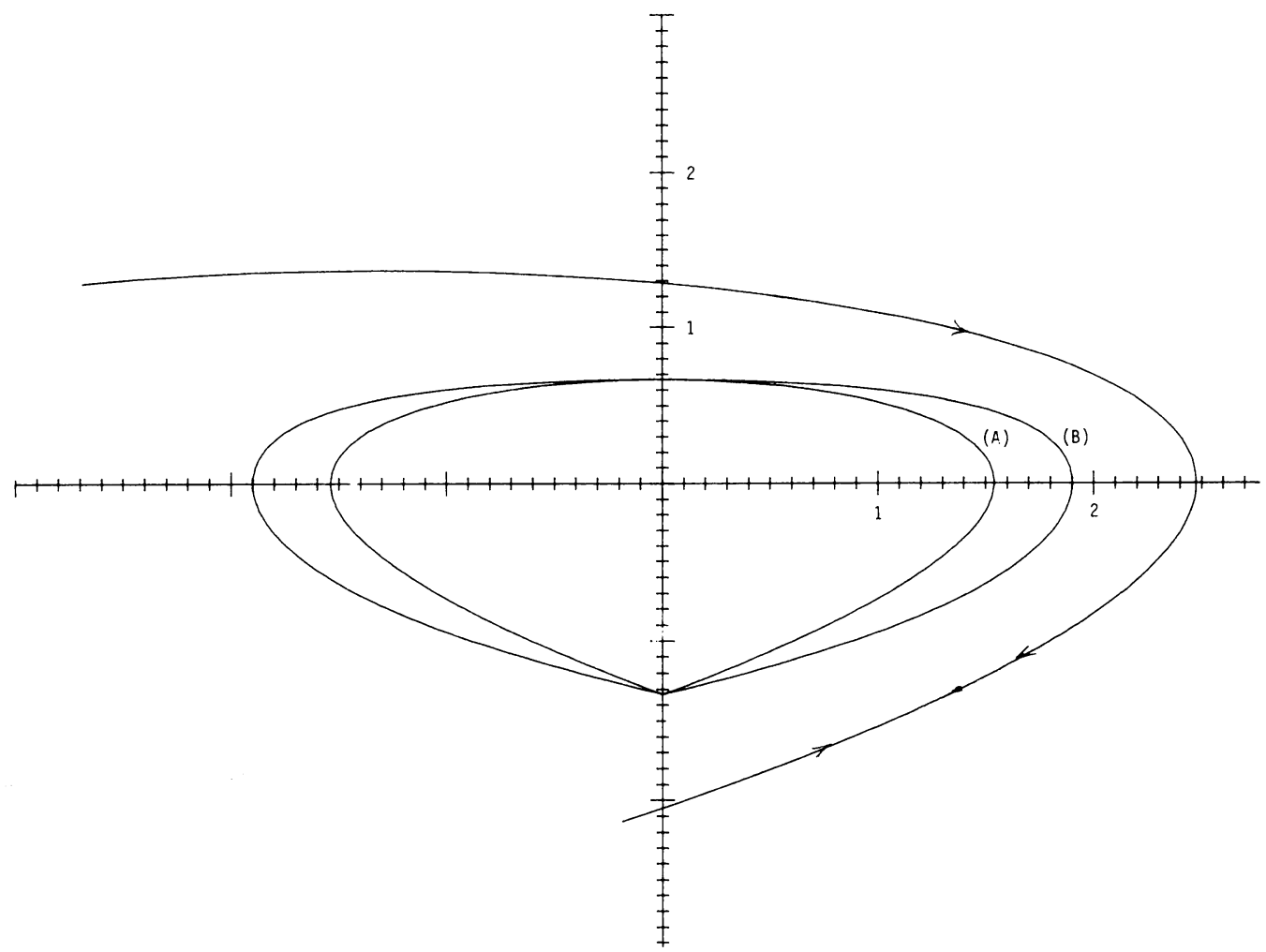

Fig. 2. (A) Curve (4.6), $b=2$; (B) curve (4.5), $a=1, b=2$. 


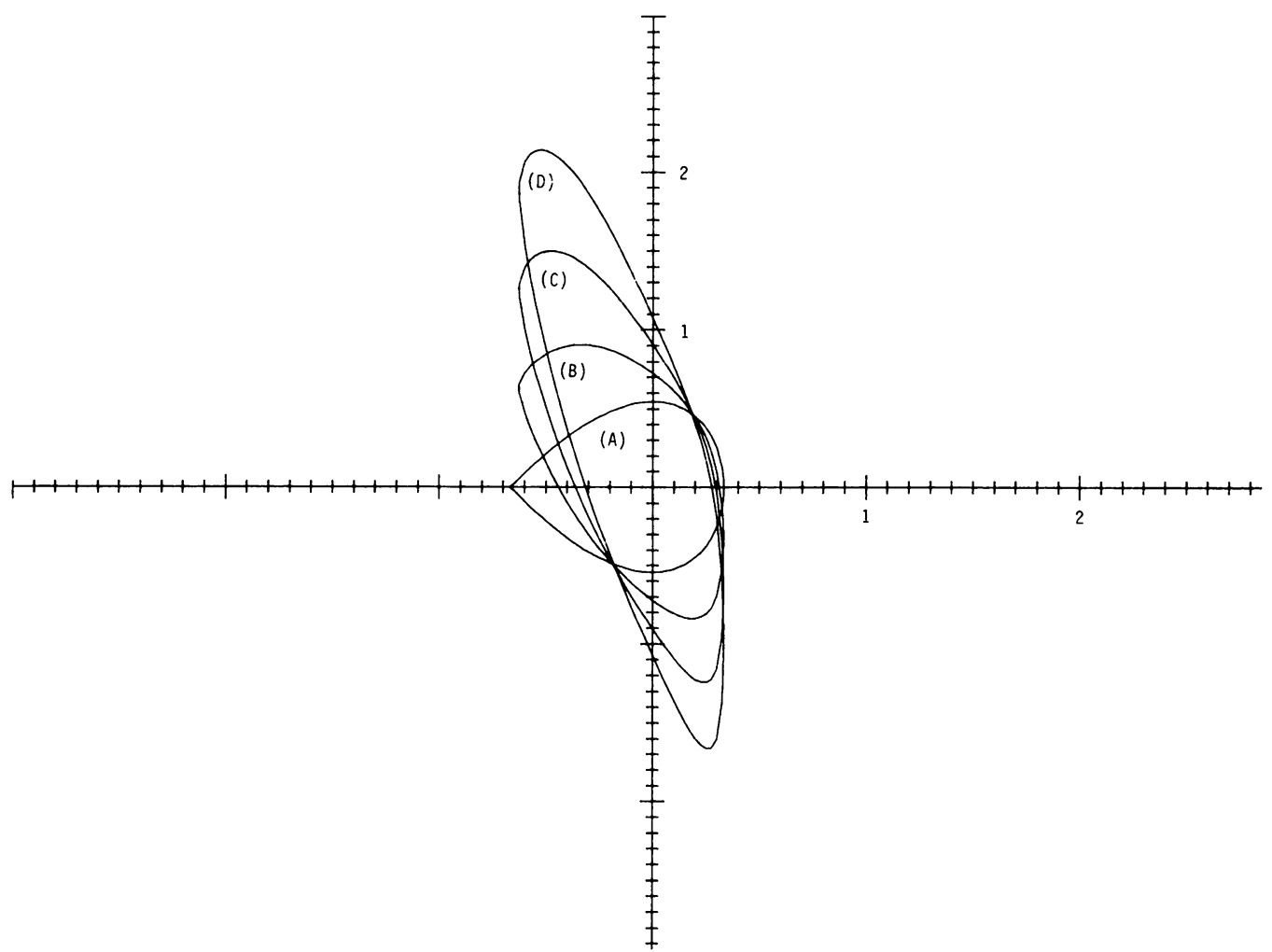

Fig. 3. (A) Curve (4.8), $b=1$; (B) curve (4.7), $a=1, b=1$; (C) curve (4.7), $a=2, b=1$; (D) curve (4.7), $a=3$, $b=1$.

Thus,

$$
\frac{\left(b y^{2}+y^{3}\right)^{2}}{2}+\lambda_{0} \frac{x^{2}}{2}\left(b y^{2}+y^{3}\right)+\lambda_{0} \frac{x^{4}}{8}=\left[\frac{4 b^{3}}{27}\right]^{2} \cdot \frac{1}{2} \quad\left(y \geq-\frac{2 b}{3}\right)
$$

determines the maximal region of asymptotic stability over the weight functions $\theta(x, y)=\lambda_{1}\left(b y^{2}+y^{3}\right)+\lambda_{2} x^{2} / 2$ for $(4.2)$.

From the preceding section it should be noted that (4.5) totally encloses

$$
V(x, y)=b y^{2}+y^{3}+x^{2} / 2=4 b^{3} / 27 .
$$

Indeed, it can be shown that for $b$ fixed, $\omega(\lambda, y) \simeq 2 a\left[3\left(k^{2}-I^{2}(y)\right)(2 b)^{-1}\right]^{1 / 2}$ as $a \rightarrow \infty$ for $-2 b / 3 \leq y \leq b / 3$, where $\omega(\lambda, y)$ is defined in (3.11). This is shown in Fig. 1 where $b=2$ and $a$ varies. Fig. 2 shows $V_{\theta}$ and $V$ where $a=1, b=2$ in comparison with the actual region of asymptotic stability.

It is also useful to transform (4.5) back to the phase plane and compare it with a previous choice for $V$. From (4.2), $x=-\dot{y}-a y$, so (4.5) becomes

$$
\frac{\left(b y^{2}+y^{3}\right)^{2}}{2}+\lambda_{0}\left(b y^{2}+y^{3}\right) \frac{(\dot{y}+a y)^{2}}{2}+\lambda_{0} \frac{(\dot{y}+a y)^{4}}{8}=\left[\frac{4 b^{3}}{27}\right]^{2} \cdot \frac{1}{2}
$$

in the phase plane. For (4.1),

$$
\dot{y}^{2} / 2+b y^{2}+y^{3}=4 b^{3} / 27
$$


is an estimate of the region of asymptotic stability that has been employed elsewhere (see $[3,5])$. These curves are compared in Fig. 3 where $b=1$ and $a$ varies.

\section{REFERENCES}

[1] L. Anderson, Estimates of planar regions of asymptotic stability, Quart. Appl. Math. 36, 434-438 (1979)

[2] L. Anderson and W. Leighton, Liapunov functions for autonomous systems of second order, J. Math. Anal. Appl. 23, 645-664 (1968)

[3] J. P. LaSalle and S. Lefschetz, Stability by Liapunov's direct method, with applications, Academic Press, New York, 1961

[4] W. Leighton, Morse theory and Liapunov functions, Rend. Circulo Matem., Ser. 2, 1-10 (1964)

[5] W. Leighton, On the construction of Liapunov functions for certain autonomous nonlinear differential equations, Contrib. Diff. Eqs. 2, 367-383 (1963)

[6] R. Struble, Nonlinear differential equations, McGraw-Hill Book Co., Inc., New York, 1962 